\title{
Relation Between Birth Condition and Neuro-Behavioral Organization in the Neonate
}

\author{
G.TuRKEWITZ ${ }^{[15]}$, T. MOREAU and H. G. BirGh \\ Department of Pediatrics, Albert Einstein College of Medicine, Bronx, New York, USA
}

\begin{abstract}
Extract
The neuro-behavioral organization of three groups of infants who differed in their condition at birth as determined by their Apgar score was examined during the first week of life. At the time of testing, all infants, regardless of birth status, were in good condition according to clinical evaluation. Testing consisted of the presentation of 30 lateralized somesthetic stimuli to the perioral region of each infant ( 15 to each side). The response measure used was the lateral direction of the first head turn. As a group, normal infants (Apgar scores of 9-10) were found to be more responsive to stimulation of the right than of the left, and to make more ipsilateral responses to stimulation of the right than of the left. Infants whose condition at birth was poor (Apgar scores 1-6) did not show these patterns of response but were equally likely to be preponderantly responsive to stimulation at either side. The degree of lateral differentiation was atypically small even for those infants of poor birth status who exhibited the typical direction of differentiation, i.e., who made more ipsilateral responses to stimulation of the right than of the left. Furthermore, the proportion of infants of poor birth status who made any contralateral responses was higher than the proportion of normal infants who made such responses. Results from a group of infants whose condition at birth was questionable (Apgar scores 7-8) fell between those from the extreme groups with respect to all of the analyses.
\end{abstract}

\section{Speculation}

Poor condition at birth has persistent effects on behavioral organization even in babies who, in clinical judgment, have recovered normal functioning. To the extent that early behavioral organization may be relevant to the subsequent development of lateral differentiation, these effects could be related to the association between a variety of disabilities and disturbances in lateral differentiation which has been found in older children and adults.

Introduction

The present study, which analyzes the relation between disturbances in laterally differentiated behavior and poor condition at birth, stemmed from concern with the possibility that poor condition at birth may have persistent effects on neuro-behavioral development. Frequently, infants whose condition at birth is suboptimal appear to be normal by the third day of life.
There is evidence, however, that in the group as a whole, there is a relatively high frequency of abnormalities in motor, language and intellectual functions during later infancy and childhood $[4,5,6]$. It is of course possible, though unlikely, that these later abnormalities are exhibited solely by infants who did not show rapid apparent recovery. It is more likely that infants who did exhibit rapid recovery are represented among children who later show abnormalities in func- 
tioning. It therefore appeared possible that the effects of poor condition at birth on behavioral organization were not evanescent and that the recovery usually noted during the first few days of life was more apparent than real, with continuing disorganization masked by the insensitivity of routine clinical evaluation.

To explore this possibility, the neuro-behavioral organization of infants who had been in poor condition at birth but who had apparently recovered was compared with that of infants whose condition at birth and thereafter was good. To make this comparison, it was necessary to select a measure of neuro-behavioral organization which is clearly defined and which has a characteristic form in normal infants. The directed head-turning response to lateralized somesthetic stimulation of the perioral region was selected because it satisfied these requirements. A variety of dimensions along which this response can be defined have been proposed by Prechte [9] and by Turkewitz et al. [10], and a clearly organized and consistent pattern of directed head-turning to laterally applied somesthetic stimulation has been repeatedly found in normal infants $[10,11]$.

\section{Subjects and Procedure}

Subjects

The sample consisted of 130 infants who were between 24 and 81 hours old (mean age: $58.7 \pm 15.6$ ). All infants were selected from the well-baby nurseries of the Bronx Municipal Hospital Center. At the time of testing for lateral differentiation, all of the infants were in good condition as determined by physical examination, and none was of special clinical concern. On the basis of their condition at birth, as assessed by Apgar ratings [1, 2] assigned within one minute after birth, the infants were divided into three groups: a High Apgar Group (scores of 9-10); an Intermediate Apgar Group (scores of 7-8); and a Low Apgar Group (scores of 1-6). The distributions of subjects by Apgar score and sex are presented in table I.

\section{Procedure}

Directional head-turning in response to lateralized somesthetic stimulation of the perioral region was used as the test of neuro-behavioral competence. Testing consisted of the presentation of 30 lateralized stimuli (15 to each side). Each stimulus was a touch with a Grumbacher standard round camel's hair brush. The stimulus was applied to the angle of the infant's mouth and had an average duration of one second.

The infant was placed in the supine position prior to testing and remained in that position throughout the test series. Any wrappings capable of interfering with movements of arms or legs were removed and sleeping infants were awakened before testing was begun. During testing, the subject's head was held in the midline prior to and during the application of each stimulus. The head was held at the bitemporal regions between the experimenter's thumb and finger. Stimuli were presented only when the experimenter could detect no lateral pressure against the restraining fingers. The head was released simultaneously with the removal of the stimulus. Before the application of each stimulus, the head was repositioned to the midline. The interstimulus interval varied because of differences in the time required to obtain the necessary midline balance, but rarely exceeded 40 seconds and was never less than 15 seconds. The experimenter who applied the stimuli and observed the infant's responses had no knowledge of the Apgar status of the infant being tested.

The response recorded was the direction of the first lateral head movement made from the beginning of stimulation to one second following its termination. Any such head movement, regardless of magnitude, was scored as a response. Since we were concerned with direction rather than with either latency or magnitude of head-turning, an automated device for measuring head-turning responses was not. used. A check on the inter-observer reliability in determining the occurrence and direction of head-turning responses indicated an agreement level of $94 \%$.

\section{Results}

Analysis of the data indicated that although poor condition at birth was not associated with an atypical frequency of head-turning responses, it was associated with atypical lateral differences in these responses. This association was manifested in a number of ways, each of which will be separately considered.

Table I. Distributions of subjects by Apgar score and sex

\begin{tabular}{lllll}
\hline Status & $\begin{array}{l}\text { Apgar } \\
\text { score }\end{array}$ & $\begin{array}{l}\text { Male } \\
\text { No. }\end{array}$ & $\begin{array}{l}\text { Female } \\
\text { No. }\end{array}$ & $\begin{array}{l}\text { Total } \\
\text { No. }\end{array}$ \\
\hline Low & 1 & 1 & 1 & 2 \\
& 2 & 0 & 1 & 1 \\
& 3 & 2 & 1 & 3 \\
& 4 & 2 & 4 & 6 \\
& 5 & 3 & 5 & 8 \\
& 6 & 7 & 5 & 12 \\
\hline Inter- & 7 & 3 & 4 & 7 \\
mediate & 8 & 10 & 10 & 20 \\
\hline High & 9 & 29 & 26 & 55 \\
& 10 & 10 & 6 & 16 \\
\hline & Total & 67 & 63 & 130 \\
\hline
\end{tabular}


Lateral Differences in Frequency of Response

As may be seen in table II, infants who were in the High Apgar Group were significantly more responsive to stimulation at the right than at the left. This lateral difference in responsiveness was also exhibited by subjects in the Intermediate Apgar Group. In contrast, infants in the Low Apgar Group showed no such preponderant responsiveness to stimulation at the right and in fact were slightly more responsive to stimulation at the left.

To determine the extent to which the lateral differences in the frequency of directional responding exhibited by the various groups were characteristic of the individual members, the data were analyzed in terms of the proportion of infants exhibiting various patterns of directional responsiveness. When infants were classified on the basis of the side of stimulation to which they were more responsive, three patterns were identified: (1) a greater frequency of response to stimulation of the right than of the left; (2) a greater frequency of response to stimulation of the left than of the right; and (3) an equal frequency of response to stimulation of the right and of the left. The data presented in table III indicate that in the High Apgar Group, the proportion of infants who were more responsive to stimulation at the right was significantly higher than the proportion who were more responsive to stimulation at the left. This was also the case in the Intermediate Apgar Group. No such difference was found in the Low Apgar Group. In addition, it may be noted that the proportion of infants in the Low Apgar Group who were equally responsive to stimulation of the two sides was twice that of the High Apgar Group and four times that of the Intermediate Apgar Group. These differences were significant at the 0.07 and 0.04 levels of confidence, respectively ( $\mathrm{z}$ tests for

Table II. Relation of condition at birth to lateral differences in frequency of head-turning responses

\begin{tabular}{llllll}
\hline \multicolumn{5}{c}{$\begin{array}{l}\text { Percentage of responses to } \\
\text { stimulation }\end{array}$} \\
\hline Apgar status & $\begin{array}{l}\text { At the right } \\
\text { Mean SD }\end{array}$ & $\begin{array}{l}\text { At the left } \\
\text { Mean SD }\end{array}$ & $\mathrm{t}^{1}$ \\
\hline & 65.4 & 21.67 & 66.5 & 22.40 & 0.04 \\
$\begin{array}{l}\text { Low (1-6) } \\
(\mathrm{n}=32)\end{array}$ & & & & & \\
\hline $\begin{array}{l}\text { Intermediate } \\
(7-8)(\mathrm{n}=27)\end{array}$ & 71.3 & 16.87 & 58.7 & 23.13 & $3.15^{2}$ \\
\hline $\begin{array}{l}\text { High (9-10) } \\
(\mathrm{n}=71)\end{array}$ & 71.7 & 22.27 & 58.2 & 24.60 & $6.80^{3}$ \\
\hline
\end{tabular}

${ }^{1} \mathrm{t}$ test for dependent samples ${ }^{2} \mathrm{p}<0.01{ }^{3} \mathrm{p}<0.001$ difference betweeen independent proportions, z's = 1.82 and 2.07 respectively).

\section{Relation of Direction of Response to Side of Stimulation}

When the data were analyzed for the sample as a whole, $91 \%$ of the head-turning responses were found to be ipsilateral; the other $9 \%$ were contralateral turns.

The frequency with which infants made contralateral responses was associated with condition at birth. In the Low Apgar Group, the proportion of infants making one or more contralateral head turns was 0.75 , whereas the proportions of infants in the Intermediate and High Apgar Groups making such responses were 0.52 and 0.48 , respectively. The difference between the High and Low Apgar Groups was significant at the 0.01 level of confidence ( $\mathrm{z}$ test for difference between independent proportions, $z=2.58$ ) and the difference between the Intermediate and Low Apgar Groups approached but did not reach a conventional level of statistical significance $(z=1.83 ; p=0.07)$.

\section{Degree of Lateral Differentiation}

To achieve a more refined analysis of lateral differences in the frequency of ipsilateral responses, a differentiation score was calculated for each baby. This was done by subtracting the number of ipsilateral responses to stimulation of the left perioral region from the number of such responses to stimulation of the right perioral region. A positive lateral differentiation score reflected an excess of right responses and a negative score an excess of left responses. Normal infants characteristically have positive differentiation scores.

The relation between Apgar Group and the differentiation score is presented in figure 1 . As may be seen in this figure, the mean lateral differentiation score increased as Apgar level rose. A $3 \times 1$ analysis of va-

Table III. Relation of condition at birth to proportion of infants exhibiting various patterns of lateral differences in frequency of head-turning responses

\begin{tabular}{lllll}
\hline & \multicolumn{3}{c}{ Proportion of subjects } \\
\cline { 2 - 5 } & $\begin{array}{l}\text { More responsive } \\
\text { to stimulation }\end{array}$ & $\begin{array}{l}\text { Equally responsive } \\
\text { to stimulation at } \\
\text { the right and } \\
\text { at the left }\end{array}$ \\
\cline { 2 - 4 } Apgar status & \multicolumn{4}{c}{$\begin{array}{l}\text { At the At the } \mathrm{z}^{1} \\
\text { right }\end{array}$} \\
\hline Low & 0.31 & 0.41 & 0.42 & 0.28 \\
Intermediate & 0.70 & 0.22 & $2.40^{2}$ & 0.07 \\
High & 0.76 & 0.10 & $5.90^{3}$ & 0.14 \\
\hline
\end{tabular}

1 Approximation of the binomial expansion with correction for continuity. ${ }^{2} p=0.02{ }^{8} p<0.001$ 
riance indicated that the magnitude of lateral differentiation was significantly higher in both the Intermediate and High Apgar Groups than in the Low Apgar Group $(\mathrm{F}=6.33, \mathrm{p}<0.005$; Tukey tests yielded $\mathrm{p}<0.05$ for both High versus Low and Intermediate versus Low Groups).

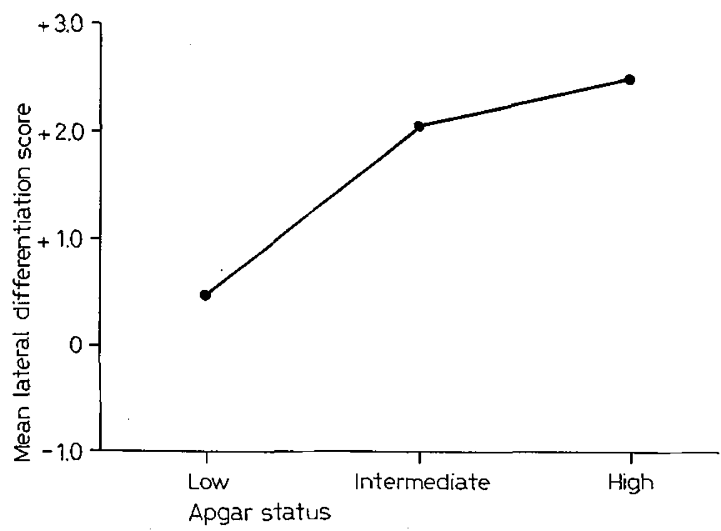

Fig.1. Relation of condition at birth to magnitude of lateral difference in frequency of ipsilateral responses.

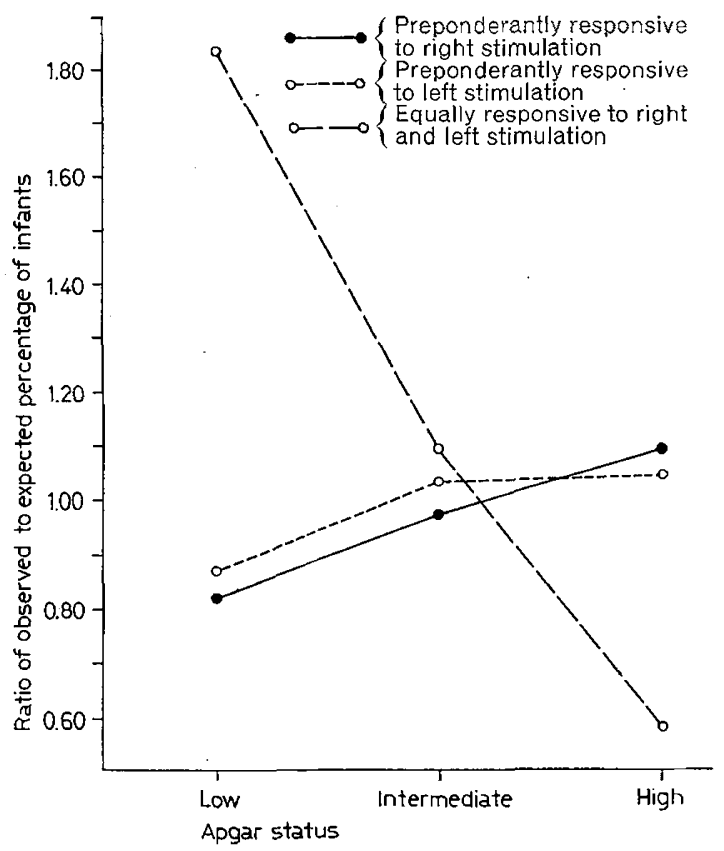

Fig. 2. Proportional representation of infants of different Apgar levels in various categories of lateral differences in frequency of ipsilateral head-turning.
Relation of Type of Differentiation to Apgar Level

The data were analyzed to determine whether the three Apgar Groups differed with respect to their representation in each of three categories of differentiation, i.e. more ipsilateral responses to stimulation of the right than of the left, more ipsilateral responses to stimulation of the left than of the right, and an equal number of ipsilateral responses to stimulation of the right and the left. If there were no relation between Apgar level and kind of differentiation, the distribution of infants of different Apgar levels in each of the ipsilateral response categories would reflect their relative size in the sample. Figure 2 shows the ratio of obtained to expected percentages of High, Intermediate and Low Apgar subjects in each of these response categories. As may be seen in this figure, the number of infants in the Low Apgar Group who were preponderantly responsive to stimulation of the left was almost twice that expected. In contrast, the number of infants in the High Apgar Group who fell into this category was only slightly more than half that expected. This difference between the two Apgar Groups was significant at the 0.05 level of confidence $\left(\chi^{2}\right.$ corrected for continuity $=4.82, \mathrm{df}=1$ ). Among the babies who were more responsive to stimulation of the right than to stimulation of the left, subjects in the High Apgar Group were somewhat overrepresented and those in the Low Apgar Group underrepresented. The distribution of subjects who made an equal number of ipsilateral responses to stimulation at the right and at the left did not appear to be systematically related to Apgar level.

The percentage of subjects in each of the Apgar Groups who exhibited the three types of lateral differentiation with respect to ipsilateral responses is pre-

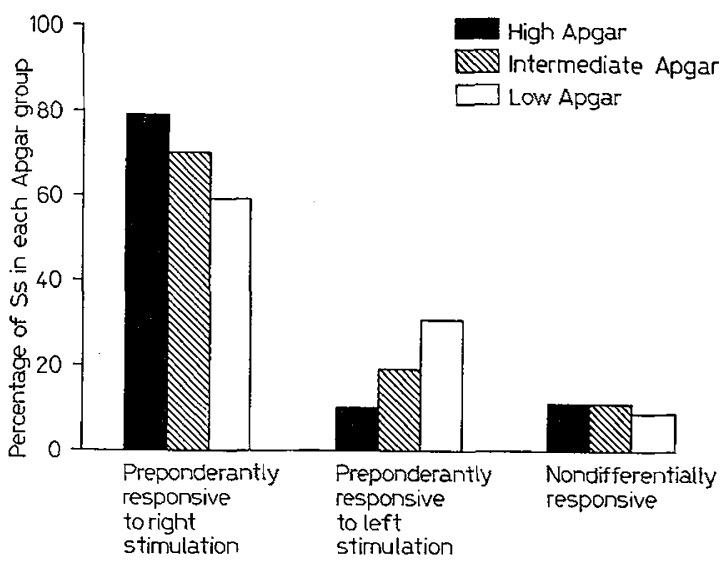

Fig.3. Relation of condition at birth to percentage of infants exhibiting various patterns of lateral differences in frequency of ipsilateral head-turning responses. 
sented in figure 3 . The percentage of subjects who were preponderantly responsive to stimulation at the right was greater in the High than in the Low Apgar Group ( $z$ test for difference between independent proportions, $z=2.05, p=0.04)$. The Intermediate Apgar Group did not differ from either of the two extreme groups with respect to the percentage of subjects who exhibited this pattern of response. Further analysis revealed that the percentage of subjects who were preponderantly responsive to stimulation of the left was higher in the Low than in the High Apgar Group $(z=2.71, p=$ 0.01). The Intermediate Apgar Group again did not differ from either of the extreme groups. As is evident in the figure, there were no significant differences between the percentage of subjects in the different Apgar Groups who made an equal number of ipsilateral responses to stimuli at the two loci.

Degree of Lateral Differentiation in Infants who Were More Responsive to Stimulation of the Right than of the Left

Since greater responsiveness to stimulation of the left than of the right results in a negative differentiation score and since relatively more infants in the Low Apgar Group had such scores, it was possible that the observed relation between magnitude of differentiation and condition at birth reflected this differential distribution of negative scores. The data were therefore reanalyzed using only those subjects who had been more responsive to right than to left stimulation. Even with this restriction, the amount of differentiation was still positively associated with Apgar level, the differentiation scores of the infants in both the Intermediate and High Apgar Groups being significantly higher than those of infants in the Low Apgar Group (MannWhitney $\mathrm{U}$ tests [12]: $\mathrm{U}=120.5, \mathrm{p}<0.05 ; \mathrm{U}=$ $360.5, z=2.09, p=0.02$, respectively). Thus, even those infants in the Low Apgar Group who in general behaved as did normal infants, showed less differentiation than did subjects in the Intermediate and High Apgar Groups.

\section{Relation Between Characteristics of the Sample and Lateral Differentiation}

Since it was possible that magnitude and type of lateral differentiation varied with sex and age at testing, the data were analyzed in relation to these variables. No differences between males and females having comparable Apgar status were found. Subjects in the present investigation ranged in age from 24 to 81 hours and infants with Low Apgar scores tended to be tested when slightly younger than were infants in the Intermediate and High Apgar Groups. The data, when analyzed with respect to age at the time of testing, failed to reveal an association between age and pattern of response for any of the groups.
Since Apgar rating is a composite based upon five different measures (heart rate, respiratory effort, muscle tone, reflex irritability, and skin color), it was possible that one or several of these measures were more closely related to neuro-behavioral organization than was the composite score. The findings indicated that each of the separate Apgar measures, with the exception of skin color, was significantly and equivalently correlated with lateral differentiation score (rho ranged from 0.20 to 0.28 ). The subscores themselves tended to be highly correlated (rho ranged from 0.50 to 0.73 ). This analysis, therefore, provides no evidence that any particular measure made a unique contribution to neuro-behavioral outcome.

The criteria used for selection of infants with High Apgar scores resulted in the exclusion of infants with pre- or perinatal complications. No such exclusions were made in the selection of infants with Low Apgar scores. The data from infants with Low Apgar scores were therefore analyzed to determine whether there was a relation between either the fact or the type of complication and subsequent lateral differentiation. Only seven of the infants had any complications noted; three of these infants were delivered via cesarean section and the remaining four had various other complications. When the data from these seven infants were compared with those from the other 25 infants with Low Apgar scores, there were no apparent differences.

It is well known that the responsiveness of infants to stimulation may be affected by their prandial condition and by the time of day at which examination is carried out. Since our infants were tested at slightly different times of day and in somewhat different relations to the time of feeding, it was necessary to compare the three groups when both prandiality and time of testing were controlled. Such an analysis resulted in no significant modification of the findings that have already been reported.

\section{Discussion}

The results clearly indicate that a group of infants who, on clinical evaluation, have apparently made a full recovery from a poor condition at birth continue to exhibit disturbances in neuro-behavioral organization during the first week of life. This group was atypical with respect to: (1) lateral differences in the frequency of head-turning responses; (2) the proportion of infants who were preponderantly responsive to stimulation at the left and the proportion who were preponderantly responsive to stimulation at the right; (3) the magnitude of lateral differentiation both when all infants 
were considered and when only those who were more responsive to stimulation at the right were considered; and (4) the proportion of infants making contralateral responses.

The finding of an association between poor condition at birth and atypical patterns of lateral differentiation in infants who had, in clinical judgment, made a normal recovery suggests either that recovery was more apparent than real or that the early malfunctioning had contributed, during the perinatal period, to a sequence of development which did not lead to the establishment of typical patterns of differentiation. The available data do not permit us to choose between these alternatives.

The finding that not all infants of poor birth status exhibited disturbed patterns of lateral differentiation also suggests several possibilities: (1) Poor condition at birth is associated in an 'all-or-none' manner with neuro-behavioral organization and affects the pattern of lateral differentiation of some infants but not of others. (2) Poor condition at birth results not only in relatively high frequencies of atypical patterns of lateral differentiation, but also in an overall change in the degree of differentiation. Support for this possibility is provided by the finding of a relation between Apgar status and magnitude of lateral differentiation even when consideration was restricted to those infants who were like normal infants with respect to the direction of lateral differentiation. (3) Poor condition at birth results in an increase in individual variability of responsiveness. Such increased variability would be congruent with results obtained by KLATSKIN et al. [6] who found that infants with Low Apgar scores ( 6 or below) were, by the age of $6-7$ months, more variable in performance when tested for adaptive behavior and for fine and gross motor behavior than were infants with higher Apgar scores (7 or above). Investigations are now in progress in our laboratory to determine whether poor birth condition is associated with greater individual variability of lateral responsiveness.

The results of the present investigation also suggest that Apgar level is associated with a continuum of dysfunction. The finding that infants in the Intermediate Apgar Group fell between those in the High and Low Apgar Groups on all of the measures utilized suggests that the differences between the infants with Intermediate Apgar scores and those having High and Low scores are real although not always statistically significant. These results are consistent with those of LEwIS et al. [7], who found differences in visual responsiveness at the age of three months between groups of infants who had perfect Apgar scores (i.e., 10) and those who had relatively high but imperfect scores (i.e., 7, 8 and 9). The present data, together with those of LEwIs et al. strongly indicate that despite its apparent crudity, the
Apgar score predicts group differences in outcome not only between infants at the two extremes of physiologic function, but also between these infants and those in the 'near normal' range.

It is tempting to speculate about the possible developmental consequences of disturbances in early lateral differentiation. Elsewhere, we have suggested that the early-appearing lateral differentiation of behavior provides 'a possible basis for the subsequent development of more far-reaching and functionally significant lateral differences... The early lateralization of responsiveness may result in the establishment of a leading side in dealing with the environment and either directly or via a learning process result in the increasing permanence of... lateral preference.' [11] It is possible that this suggested developmental sequence may be modified by the failure of early lateral differentiation to occur, and that infants who are in poor condition at birth are at this type of developmental risk. To the extent that the failure of early lateral differentiation to occur is related to the subsequent development of atypical patterns of lateral differentiation, the results of the current investigation may be related to the association among a variety of disabilities and disturbances in lateral differentiation which has been found in older children and adults $[3,8]$.

\section{Conclusions}

The findings of this study lead to two general conclusions. (1) Poor condition at birth has persistent although subtle effects which are detectable through the use of a sufficiently sensitive measure. (2) The nature of these effects may be relevant to the disordered development of lateral differentiation.

\section{References and Notes}

1. Apgar, V.; Holaday, D. A.; James, L.S.; WeisBRot, I. M. and BERrien, C.: Evaluation of the newborn infant: Second report. J.amer. med. Ass. 168: 1985 (1958).

2. Apgar, V. and James, L. S.: Further observations on the newborn scoring system. Amer.J. Dis. Child. 104: 419 (1962).

3. BraIN, W.R.: Speech and handedness. Lancet 249: 837 (1945).

4. EDWARDs, N.: The relationship between physiological factors immediately after birth and mental and motor performance at four years. Paper read at 38th Annual Meeting of the Eastern Psychological Association, Boston, April (1967). 
5. Graham, F.K.; Ernhart, G.B.; Thurston, D. and CRAFT, M.: Development three years after perinatal anoxia and other potentially damaging newborn experiences. Psychol. Monogr. 76: Whole no. 522 (1962).

6. Klatskin, E.H.; MaGarRy, M.E. and Steward, M.S.: Variability in developmental test patterns as a sequel of neonatal stress. Child Develop. 37: 819 (1966).

7. Lewis, M.; Bartels, B.; Campbell, H. and GoldBERG, S. : Individual differences in attention: The relation between infants' condition at birth and attention distribution within the first year. Amer. J.Dis. Ghild. 113: 461 (1967).

8. NAIDOo, S.: An investigation into some aspects of ambiguous handedness; MA Thesis, Univ. of London (1961).

9. Prechte, H.R.F.: The directed head-turning response and allied movements of the human baby. Behaviour 13: 212 (1958).

10. TurkewtTz, G.; Gordon, E.W. and Brrah, H. G.: Head turning in the human neonate: Effect of prandial condition and lateral preference. J. comp. physiol. Psychol. 59: 189 (1965).

11. Turkewitz, G.; Moreau, T.; Birch, H.G. and Grystal, D.: Relationship between prior head position and lateral differences in responsiveness to somesthetic stimulation in the human neonate. J. exp. Ghild Psychol. 5: 548-561 (1967).

12. A nonparametric test was used because the omission of nonpositive scores resulted in distributions which were not normal. One-tailed tests were used because the question being asked was unidirectional, i.e., would the subjects with Low Apgar scores continue to show a lesser degree of lateral differentiation when the depression of the mean stemming from negative scores was eliminated?

13. The authors thank Mrs. Linda Davis and Mr. David Grystal for their assistance and Miss WINTERs and the nurses of the Well Baby Unit of the Bronx Municipal Hospital Center for their help and cooperation.

14. This investigation was supported in part by the National Institutes of Health, National Institute of Child Health and Human Development (HD00719); by the Association for the Aid of Crippled Children; and by the National Association for Retarded Children.

15. Requests for reprints should be addressed to: Gerald Turkewitz, Ph.D., Department of Pediatrics, Albert Einstein College of Medicine, Eastchester Road, Bronx, N.Y. 10461 (USA). 